\title{
Liquid water infiltration into a layered snowpack: evaluation of a 3-D water transport model with laboratory experiments
}

\author{
Hiroyuki Hirashima ${ }^{1}$, Francesco Avanzi ${ }^{2, a}$, and Satoru Yamaguchi ${ }^{1}$ \\ ${ }^{1}$ Snow and Ice Research Center, National Research Institute for Earth Science and Disaster Resilience, \\ Suyoshi-machi, Nagaoka-shi, Niigata-ken, 940-0821, Japan \\ ${ }^{2}$ Department of Civil and Environmental Engineering, Politecnico di Milano, Milan, Italy \\ ${ }^{a}$ now at: Department of Civil and Environmental Engineering, University of California, Berkeley, Berkeley, USA \\ Correspondence to: Hiroyuki Hirashima (hirasima@bosai.go.jp)
}

Received: 4 April 2017 - Discussion started: 2 May 2017

Revised: 21 September 2017 - Accepted: 21 September 2017 - Published: 10 November 2017

\begin{abstract}
The heterogeneous movement of liquid water through the snowpack during precipitation and snowmelt leads to complex liquid water distributions that are important for avalanche and runoff forecasting. We reproduced the formation of capillary barriers and the development of preferential flow through snow using a three-dimensional water transport model, which was then validated using laboratory experiments of liquid water infiltration into layered, initially dry snow. Three-dimensional simulations assumed the same column shape and size, grain size, snow density, and water input rate as the laboratory experiments. Model evaluation focused on the timing of water movement, thickness of the upper layer affected by ponding, water content profiles and wet snow fraction. Simulation results showed that the model reconstructs relevant features of capillary barriers, including ponding in the upper layer, preferential infiltration far from the interface, and the timing of liquid water arrival at the snow base. In contrast, the area of preferential flow paths was usually underestimated and consequently the averaged water content in areas characterized by preferential flow paths was also underestimated. Improving the representation of preferential infiltration into initially dry snow is necessary to reproduce the transition from a dry-snow-dominant condition to a wet-snow-dominant one, especially in long-period simulations.
\end{abstract}

\section{Introduction}

The heterogeneous movement of liquid water through the snowpack during precipitation and snowmelt leads to complex liquid water distributions that impact the snow structure through wet snow metamorphism. Furthermore, grain growth and subsequent changes in pore sizes and pore size distribution under wet conditions decrease snow strength (Wakahama, 1968; Raymond and Tsushima, 1979; Colbeck, 1983; Brun and Ray, 1987; Marsh, 1987; Brun et al., 1989; Lehning et al., 2002; Yamanoi and Endo, 2002; Ito et al., 2012) and can lead to wet snow avalanches (Kattelmann, 1984; Fierz and Föhn, 1994; Baggi and Schweizer, 2008; Mitterer et al., 2011; Mitterer and Schweizer, 2013; Takeuchi and Hirashima, 2013; Wever et al., 2016a). Liquid water movement through the snowpack also controls the lag between rain events or snowmelt and water arrival at the snow base.

In the early theories of liquid water movement, capillary gradients in snow were usually neglected (Colbeck, 1972; Colbeck and Davidson, 1972; Colbeck, 1974a, b, 1976; Dunne et al., 1976; Wankiewicz, 1978). For example, Marsh and Woo (1985) developed a model of flow channels but neglected the gradient term of capillary pressure. A twodimensional (2-D) model by Illangasekare et al. (1990) considered the gradient of capillary pressure, but focused on the effects of ice layers without considering the dependency of capillary pressure on grain size and density. A 2-D model by Daanen and Nieber (2009) adopted a van Genuchten model with dependence on grain size. For each of these models, the main cause of heterogeneous water movement was attributed to refreezing and ice layers. In porous media (e.g. soil), wa- 
ter can pond owing to capillary barriers, which consequently delays infiltration (e.g. Clifford and Stephen, 1998; Kämpf et al., 2003); It has been observed that water can pond and consequently form preferential flow in layered snow even when no ice layer is present (Waldner et al., 2004; Eiriksson et al., 2013; Katsushima et al., 2013; Avanzi et al., 2016).

Capillary barriers form owing to differences in the matrix potential between layers. Hirashima et al. (2010) replicated capillary barrier formation in the SNOWPACK model using parameters of matrix potential obtained from gravity drainage column experiments performed by Yamaguchi et al. (2010). Wever et al. (2014) incorporated the Richards equation into the SNOWPACK model and obtained a good correlation with observed runoff. Wever et al. (2015) compared upGPR data with lysimeter data and showed that, even if the simulated waterfront did not arrive at the snow base, runoff was still initiated. This was interpreted as reflecting the effect of preferential flow, which was not included in the model (Wever et al., 2015).

More recent studies have explicitly modelled preferential flow; for example, Katsushima et al. (2013) used laboratory experiments in vertically homogeneous snow to show that water entry suction, which in turn is related to grain size, affects the formation of preferential flow. On the basis of this work, Hirashima et al. (2014a) developed a threedimensional (3-D) water transport model for snowpacks that is able to reproduce preferential flow as a function of water entry suction, and validated it using the results of Katsushima et al. (2013). However, as snowpacks typically contain multiple layers of snow with different densities and grain sizes, simulations and laboratory experiments of water infiltration for different snow layers remain necessary. Furthermore, because simulation results for layered snow have not yet been validated using real data (Hirashima et al., 2013, 2014b), the accuracy of the model remains uncertain. Avanzi et al. (2016) performed infiltration experiments for multi-layered snowpacks with different combinations of grain size and infiltration rate and measured liquid water distribution, thickness of the capillary barrier, and arrival time. In this study, simulations of liquid water infiltration into layered snowpacks were performed by reproducing the laboratory experiments of Avanzi et al. (2016). The purpose of this study was (1) to evaluate the accuracy of a 3-D water transport model in reproducing infiltration patterns in layered snow; (2) to gain further insight into the 3-D infiltration process into layered snow by comparing simulation results with data from laboratory experiments; and (3) to identify future avenues of development for 3-D water transport schemes in snow.

Recently, a dual domain approach has been suggested to consider preferential flow effects in 1-D (Wever et al., 2016b; Würzer et al., 2017). Similarly, Leroux and Pomeroy (2017) developed a 2-D water transport model based on the scheme of Hirashima et al. (2014a), but considering melt-freeze processes. Reproducing heterogeneous processes in a 1-D or 2$\mathrm{D}$ model requires several assumptions. In natural snow, wa- ter flow shows lateral spreading, especially at capillary barriers, which creates complex 3-D stratigraphic features at a grain/layer scale. Furthermore, when 3-D preferential flow paths form in dry snow, wet snow area is proportional to the square of preferential flow size and inversely proportional to the square of the distance between paths (see Fig. S1 in the Supplement). For a 2-D simulation, wet snow area is e.g. proportional to preferential flow size and inversely proportional to the distance between paths (see Fig. S1). Considering a 3-D geometry can, therefore, help to define the necessary parameterizations of preferential flow effects needed to inform models with a reduced number of dimensions. Note that, while Leroux and Pomeroy's model also includes temperature and melt-freeze processes, this is not expected to play a role here as the validation experiments were performed under isothermal conditions.

\section{Simulation method}

\subsection{Model}

Details of the multi-dimensional water transport model are provided in Hirashima et al. (2014a). Models of liquid water movement in porous media use the Richards equation and the Darcy-Buckingham law, which require knowledge of capillary pressure gradients and hydraulic conductivity. However, while the equation parameters depend on porosity, pore shape, pore connectivity, size distribution, and tortuosity, they are frequently estimated from a combination of snow density and grain size (Jordan et al., 2008).

In the 3-D model used here (Hirashima et al., 2014a), the relationship between capillary pressure, water content, grain size, and snow density (the so-called water retention curve) was determined based on gravity drainage column experiments performed by Yamaguchi et al. (2012). The relationship between saturated hydraulic conductivity, snow density, and grain size was estimated from the results of Calonne et al. (2012), who considered snow microstructure using the equivalent sphere radius estimated from specific surface area (SSA, instead of grain size). We considered grain size to be equal to equivalent sphere radius (Hirashima et al., 2014a) assuming grain shapes are round. If the grain shape is dendritic, an alternative method to estimate saturated hydraulic conductivity is necessary, including a simulation of SSA (Carmagnola et al., 2014). Unsaturated hydraulic conductivity was estimated using the van Genuchten-Mualem model (Mualem, 1976; van Genuchten, 1980). Water entry suction, which is necessary to reproduce preferential flow (Hirashima et al., 2014a), was measured and formulated as a function of grain size following the approach of Katsushima et al. (2013). 


\subsection{Comparative simulation}

Hirashima et al. (2014a) performed infiltration simulations within columns with only one layer of snow. A number of multi-layer simulations were also tested (Hirashima et al., 2013, 2014b); however, they were performed in 2-D and were not validated with observations. In this study, validation of the water transport model for layered snow was performed using observations of infiltration patterns performed using dye trace experiments (Avanzi et al., 2016). In these experiments, snow samples were prepared in a cold room at $-20^{\circ} \mathrm{C}$ using refrozen melt forms. Snow was packed in a cylindrical container composed of several acrylic rings; the height and diameter of the rings were 20 and $50 \mathrm{~mm}$, respectively. Each sample was composed of two layers: the upper layer was $10 \mathrm{~cm}$ thick, and the lower layer was either 8 or $10 \mathrm{~cm}$ thick (see Avanzi et al., 2016). Then, samples were moved to a second cold room at $0^{\circ} \mathrm{C}$ and stored for at least $12 \mathrm{~h}$ to reach initial conditions of dry snow at $0^{\circ} \mathrm{C}$. All samples were characterized by a finer-over-coarser layering (i.e. the upper layer was created using a smaller grain size than the lower one), which aimed to reproduce capillary barriers. The three classes of snow grain size included fine $(0.25-0.5 \mathrm{~mm})$, medium (1.0-1.4 mm), and coarse (2.0-2.8 mm). While this definition is convenient for the scope of this study, it is not consistent with the International Classification proposed by Fierz et al. (2009). Three water input rates were considered: 10,30 , and $100 \mathrm{~mm} \mathrm{~h}^{-1}$. In total, nine experiments were performed (i.e. one for each grain size/input rate combination).

The 3-D simulations had dimensions of 5, 5, and $20 \mathrm{~cm}$ in the $x, y$, and $z$ directions, respectively. The voxels were $5 \mathrm{~mm}$ on the sides. Voxels of more than $2.5 \mathrm{~cm}$ from the central axis were treated as an impermeable wall, which ensured that the simulated shape was columnar. Snow densities, grain sizes, and rates of water supply were set to the same values as in the laboratory experiments. Grain size distributions were not measured: instead, for fine and medium snow we used the median grain sizes obtained by Katsushima et al. (2013) using the same sieves $(0.41$ and $1.5 \mathrm{~mm}$, respectively). Grain size for coarse snow was determined assuming it to be 2 times the median medium grain size $(2.9 \mathrm{~mm})$. Note that grain size is expressed in two-digit accuracy, but the simulations were performed using a four-digit accuracy, as in Avanzi et al. (2016). As a measurement of horizontal structural heterogeneity, we estimated the standard deviation following the approach of Hirashima et al. (2014a), who estimated that the standard deviation of grain size is $20 \%$ of the median grain size (Katsushima et al., 2013). In this simulation, heterogeneity of snow density was not provided. As with the cases of laboratory experiments, grain size combinations in the simulation were fine-over-coarse snow (FC), fine-over-medium snow (FM), and medium over coarse snow (MC). Values of snow density and water supply rates are shown in Table 1.
Table 1. Experimental conditions following Avanzi et al. (2016).

\begin{tabular}{lrrr}
\hline $\begin{array}{l}\text { Sample } \\
\text { ID }\end{array}$ & $\begin{array}{r}W \\
\left(\mathrm{~mm} \mathrm{~h}^{-1}\right)\end{array}$ & $\begin{array}{r}\rho_{\text {DU }} \\
\left(\mathrm{kg} \mathrm{m}^{-3}\right)\end{array}$ & $\begin{array}{r}\rho_{\text {DL }} \\
\left(\mathrm{kg} \mathrm{m}^{-3}\right)\end{array}$ \\
\hline FC1 & 11.9 & 417 & 465 \\
FC2 & 28 & 449 & 483 \\
FC3 & 113 & 433 & 470 \\
FM1 & 11.9 & 444 & 484 \\
FM2 & 27.7 & 442 & 487 \\
FM3 & 110 & 455 & 510 \\
MC1 & 11 & 472 & 487 \\
MC2 & 27.3 & 498 & 480 \\
MC3 & 111 & 494 & 478 \\
\hline
\end{tabular}

The evaluation of simulations focused on the thickness of the ponding layer at the textural interface, on the liquid water distribution, on the wet snow fraction at different heights, and on different timings that are relevant for liquid water movement in snow. These include water arrival at the interface between layers, breakthrough of preferential flow in the lower layer, and arrival of liquid water at the sample base. Data of liquid water content, wet snow fraction, and thickness of the ponding layer were measured by Avanzi et al. (2016), whereas timings were obtained from available video recordings of the experiments. A small difference (mean of $0.5 \mathrm{~min}$, maximum of $3 \mathrm{~min}$ for $\mathrm{FC1}$ ) was found between the arrival times from video recordings and those in Avanzi et al. (2016); data from videos were used here for consistency with the other timings (see Table 2). The simulated timings of water arrival at the interface, entering the lower layer, and arrival at the snow base refer to the lowest elements in the upper layer, the top three elements in the lower layer, and the lowest elements of the sample, respectively. The water content in the top three elements of the lower layer was used to determine the timing of the breakthrough because preferential flow began immediately after the water content of one of these elements became larger than zero.

\section{Simulation results}

\subsection{Water percolation through preferential flow paths and capillary barrier}

Some images of the development of capillary barriers and preferential flow for FC1 are shown in Fig. 1. These figures show the front surfaces $20 \mathrm{~s}$ after the beginning of the experiment (a and e), at the arrival time of water at the interface between layers ( $b$ and $f$ ), at the time of breakthrough of preferential flow into the lower layer (c and g) and at the arrival time at the snow base ( $\mathrm{d}$ and $\mathrm{h}$ ). The simulation results showed faster than measured arrival of water at the boundary (Table 2), which implied an overestimation of vertical velocity in the model's preferential flow for this exper- 
Table 2. Timings of infiltration in laboratory experiments and simulations. All the timings were estimated from the video recording of the experiment. The "-" represents points where timings could not be estimated from the video recording.

\begin{tabular}{|c|c|c|c|c|c|c|}
\hline \multirow[b]{2}{*}{$\begin{array}{l}\text { experiment } \\
\text { ID }\end{array}$} & \multicolumn{3}{|c|}{ experiment } & \multicolumn{3}{|c|}{ simulation } \\
\hline & $\begin{array}{l}\text { arrival at } \\
\text { boundary }\end{array}$ & $\begin{array}{r}\text { preferential flow } \\
\text { formation }\end{array}$ & $\begin{array}{l}\text { arrival at } \\
\text { snow base }\end{array}$ & $\begin{array}{l}\text { arrival at } \\
\text { boundary }\end{array}$ & $\begin{array}{r}\text { preferential flow } \\
\text { formation }\end{array}$ & $\begin{array}{l}\text { arrival at } \\
\text { snow base }\end{array}$ \\
\hline $\mathrm{FC} 1$ & 34.8 & 85.0 & 89.0 & 16.7 & 79.0 & 79.7 \\
\hline $\mathrm{FC} 2$ & 15.2 & 48.5 & 49.8 & 8.7 & 38.7 & 39.0 \\
\hline FC3 & 7.1 & 12.3 & 14.0 & 4.0 & 11.7 & 12.0 \\
\hline FM1 & 20.0 & 79.0 & 89.5 & 17.0 & 72.3 & 109.0 \\
\hline FM2 & 11.3 & 33.3 & 39.8 & 10.7 & 37.3 & 58.3 \\
\hline FM3 & 6.7 & 11.2 & 13.0 & 4.3 & 11.3 & 15.7 \\
\hline MC1 & 5.3 & - & 9.5 & 9.0 & 11.0 & 11.7 \\
\hline $\mathrm{MC} 2$ & 3.0 & 5.0 & 8.0 & 4.7 & 5.3 & 5.3 \\
\hline MC3 & 0.8 & 2.5 & 4.5 & 1.7 & 1.7 & 1.7 \\
\hline
\end{tabular}

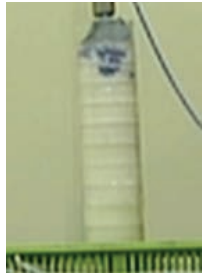

(a)
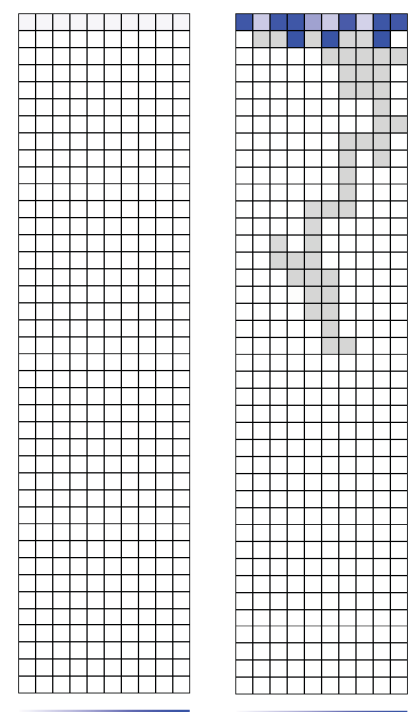

0

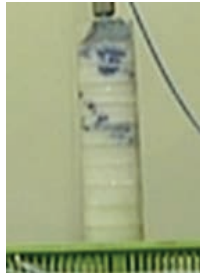

(b)

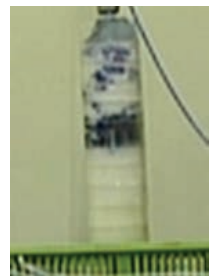

(c)

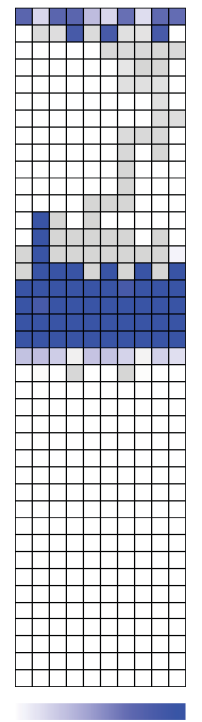

$\theta_{\mathrm{v}}$

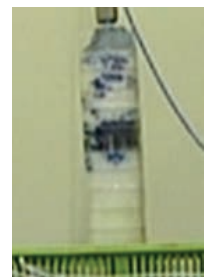

(d)
口 : Front elements

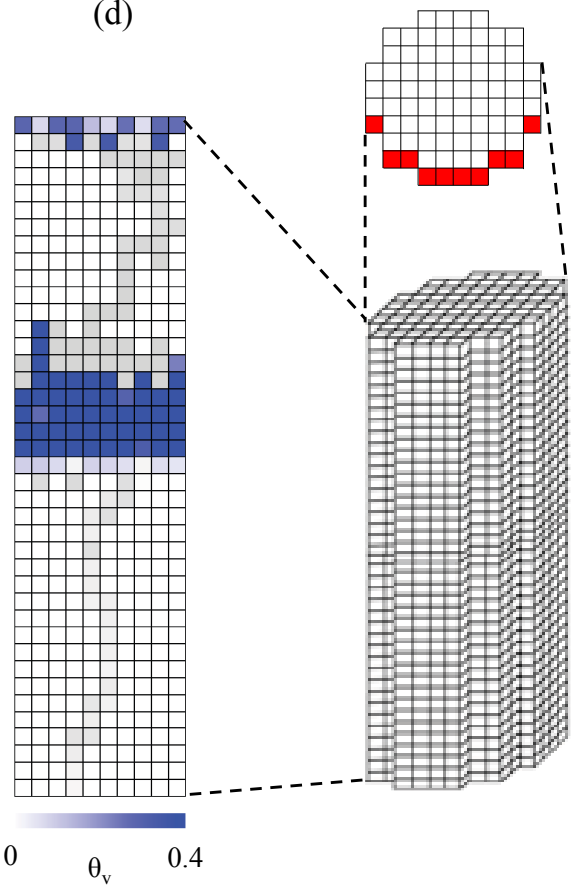

(e)

(f)

$(\mathrm{g})$

(h)

Figure 1. Development of the capillary barrier and preferential flow path for FC1 during experiments (a-d) and simulations (e-h). A blue dye tracer was used in the experiment. In the simulation images, blue denotes the liquid water content at the front elements (see the right figure), while grey denotes that the front elements are dry, but some liquid water is present within the sample at that position. The grey scale represents the maximum liquid water content for each location. Captured times were at (a) $20 \mathrm{~s}$, (b) $35 \mathrm{~min}$, (c) $1 \mathrm{~h} 25 \mathrm{~min}$, (d) $1 \mathrm{~h} 29 \mathrm{~min}$, (e) $20 \mathrm{~s}$, (f) $17 \mathrm{~min}$, (g) $1 \mathrm{~h} 19 \mathrm{~min}$, and (h) $1 \mathrm{~h} 20 \mathrm{~min}$. 
iment (Fig. 1). In Fig. $1 \mathrm{~b}$ and f, elapsed times were indeed 35 and $17 \mathrm{~min}$ in the laboratory experiment and simulation, respectively. One possible cause is the underestimation of the area of preferential flow path, which was also considered by Hirashima et al. (2014a). A smaller path area would increase conductivity because liquid water would be more concentrated and push water towards the boundary faster. After arrival at the boundary, we found that liquid water ponded above the boundary owing to a capillary barrier. In images from just before the formation of preferential flow in the lower layer (Fig. 1c and g), the elapsed time was $85 \mathrm{~min}$ in the laboratory experiment and $79 \mathrm{~min}$ in the simulation (a relative difference of $7 \%$ of the measurement value: i.e. in good agreement).

The size of laboratory experiments was restricted by the time needed to prepare and perform each of them. Also, the diameter of samples was consistent with the thickness of similar experiments in soils (see e.g. Hill and Parlange, 1972), whereas Avanzi et al. (2017a) showed that preferential flow may be intrinsically coupled with wet snow metamorphism at grain scale. This suggests that small-scale experiments are appropriate for understanding the physics of this process in snow. Nonetheless, the relatively small scale of these experiments may introduce some domain size effect. In natural snow, water flow shows lateral spreading, especially at capillary barriers, whereas experiments with small sizes may partially perturb the natural flow on snow and therefore change vertical flow owing to artificial edges. This may increase the ratio of preferential flow path area, decrease the arrival time at the base, and decrease the natural ponding amount at the capillary barrier. In terms of comparison between simulations and experiments, this effect was offset by using the same domain conditions.

The times of liquid water arrival at the base following the formation of preferential flow through the lower layer were 4 and $1 \mathrm{~min}$ in the laboratory experiment and simulation, respectively. On this basis, we calculated the propagation rate of the preferential flow path to be 0.4 and $1.6 \mathrm{~mm} \mathrm{~s}^{-1}$ for the laboratory experiment and simulation, respectively.

In the other experiments, the temporal dynamics of preferential flow formation and water ponding at the interface were generally well reproduced (Table 2; Fig. 2). The root mean square error (RMSE), the slope of a regression line with intercept equal to 0 , and the correlation coefficient $\left(r^{2}\right)$ between the simulated and measured timings were $7.8 \mathrm{~min}$, 0.97 and 0.93 , respectively. As timings were measured using frontal movies, we were sometimes unable to evaluate the timing of preferential flow formation within a sample. For example, in the case of $\mathrm{MC} 1$, preferential flow formed on the side of the sample that was not visible from the frontal position of the camera. Thus, the frontal movie did not show the preferential flow path in the lower layer, whereas horizontal spreading of water at the sample base allowed us to detect the arrival time with a reasonable precision. It follows that for this sample we cannot determine the timing of preferen-

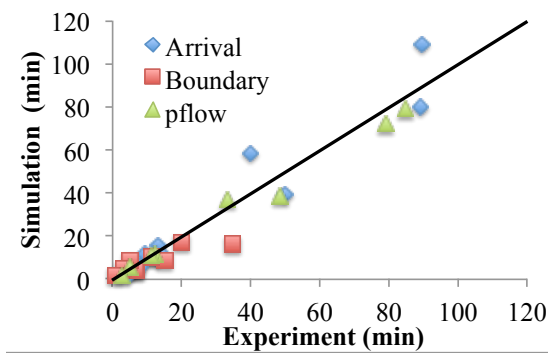

Figure 2. Timings of water arrival at the interface between layers (red squares), formation of a preferential flow path (green triangles), and arrival at the snow base (blue diamonds) for experiments and simulations.

tial flow initiation. Therefore, estimated timings from laboratory experiments may contain a delay. Overall, simulated and measured timings were consistent, which confirms that if snow parameters (e.g. snow density and grain size) are known, the arrival time of liquid water can be predicted using this model.

\subsection{Thickness of the water ponding layer}

Avanzi et al. (2016) measured the thickness of the upper layer affected by ponding at the end of each experiment. In their results for FC and FM experiments, the volumetric liquid water content on the layer boundary was about 33 to $36 \%$ ( $2 \mathrm{~cm}$ vertical resolution). The volume of ponded water was smaller for MC experiments. Laboratory experiments also showed that the thickness of the water ponding layer is not strongly connected to the water input rate. In our simulations, the influence of water input rate on the thickness of the water ponding layer was also small; however, the influence of grain size was significant (Table 3 ). The thickness of ponded water at the interface was well reproduced for the FC experiments, but was overestimated for FM experiments and underestimated for MC experiments. For MC experiments, up to $1 \mathrm{~cm}$ of ponding was shown in laboratory experiments, while simulated results showed a thickness of less than $0.5 \mathrm{~cm}$.

\subsection{Horizontal cross section}

During laboratory experiments, Avanzi et al. (2016) measured wet snow fractions at the boundary between consecutive rings using photos of the top surface of the ring below the boundary. Samples were likely slightly compressed during experiments owing to increased densification caused by wetting (Marshall et al., 1999), even though this was not noticeable. Because the model does not include settling, we chose to compare data with simulations of the inferior surface of the ring above the boundary, which returned more consistent results. At the interface between layers, most of the area of each section was wet, except for the medium over coarse samples (Fig. 3). For the other sections, only a fraction was found to 
Table 3. Thickness of the upper layer affected by ponding at the layer boundary. For each pixel at the interface between layers, simulated thickness was first determined by computing the number of voxels above with a liquid water content (LWC) of $>10 \%$. These data were then used to calculate a mean value and its standard deviation.

\begin{tabular}{lrr}
\hline $\begin{array}{l}\text { Sample } \\
\text { ID }\end{array}$ & $\begin{array}{r}\text { Experiment } \\
(\mathrm{cm})\end{array}$ & $\begin{array}{r}\text { Simulation } \\
(\mathrm{cm})\end{array}$ \\
\hline FC1 & $2-3$ & $2.8( \pm 0.5)$ \\
FC2 & $3-4$ & $3.3( \pm 0.5)$ \\
FC3 & $2-3$ & $4.1( \pm 0.4)$ \\
FM1 & $2-3$ & $4.0( \pm 0.3)$ \\
FM2 & $2-3$ & $5.1( \pm 0.3)$ \\
FM3 & $1-2$ & $4.8( \pm 0.4)$ \\
MC1 & $0-1$ & 0.1 \\
MC2 & $1-1$ & 0.3 \\
MC3 & $0.5-1$ & 0.3 \\
\hline
\end{tabular}

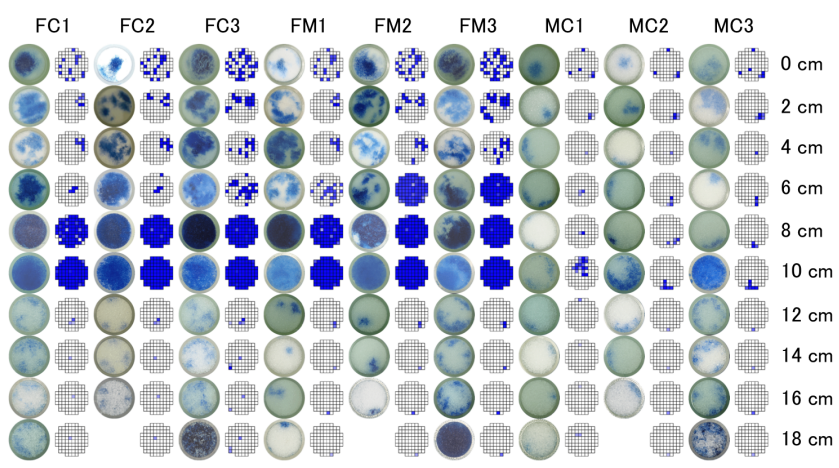

Figure 3. Liquid water distribution (blue shading) at the end of each experiment and simulation. The coordinate on the right denotes the depth of the section from the top surface.

be wet. This pattern was well simulated (Fig. 4), although simulated wet snow areas were smaller than those measured, especially in areas characterized by preferential flow. Similar underestimation by the model was also observed by Hirashima et al. (2014a).

\subsection{Water content distribution}

Our simulations were performed with $5 \mathrm{~mm}$ voxels. Simulated water contents from all voxels at a given height were averaged to obtain the water content profile. In laboratory experiments, water content profiles were obtained with a resolution of $2 \mathrm{~cm}$ (Fig. 5). The results showed that for the FC and FM experiments, the liquid water content was overestimated near the interfaces between snow layers in the upper fine layer but underestimated in other areas. The impact of water supply rate on the water content in capillary barriers was small in both simulations and experiments. Overall, simulations and observations showed good agreement in that liquid water content increased with depth in the finer layer,

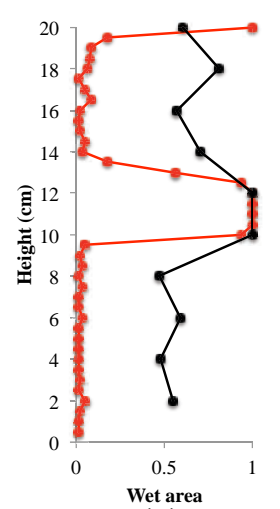

(a)

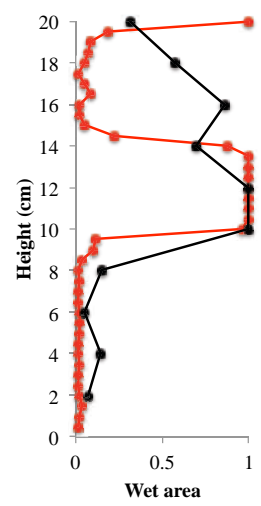

(d)

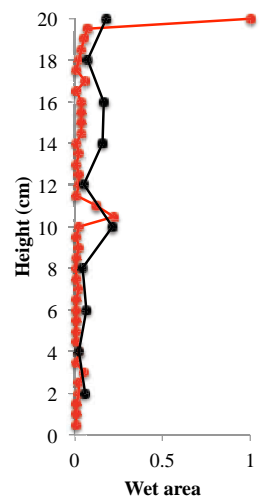

(g)

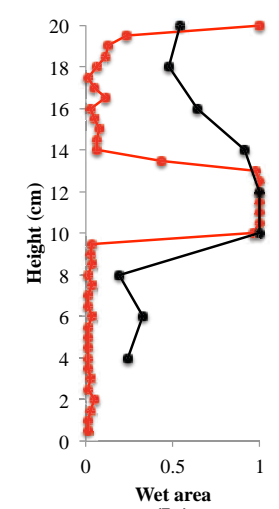

(b)

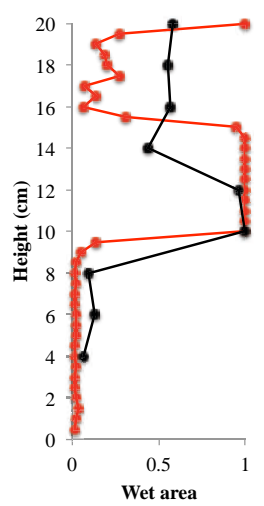

(e)

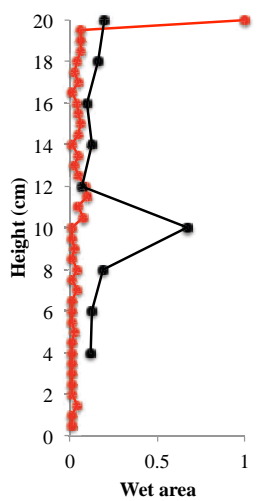

(h)

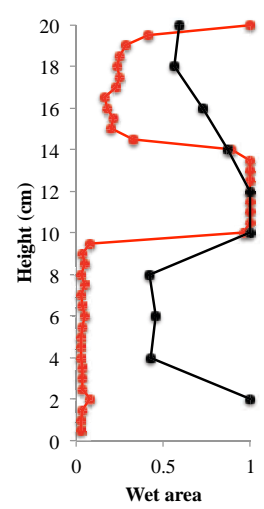

(c)

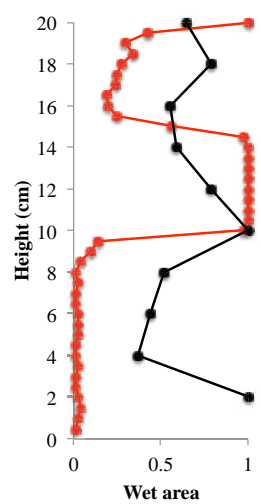

(f)

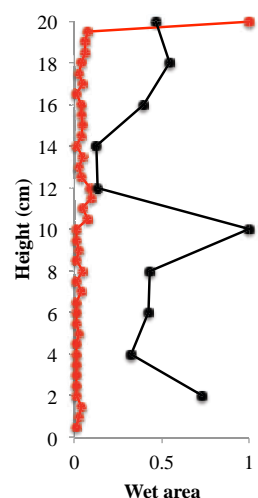

(i)
Figure 4. Profiles of wet area for experiments (red line) and simulations (black line): (a) FC1, (b) FC2, (c) FC3, (d) FM1, (e) FM2, (f) $\mathrm{FM} 3$, (g) MC1, (h) MC2, and (i) MC3.

peaked at the interface between layers, and decreased in the lower layer. 


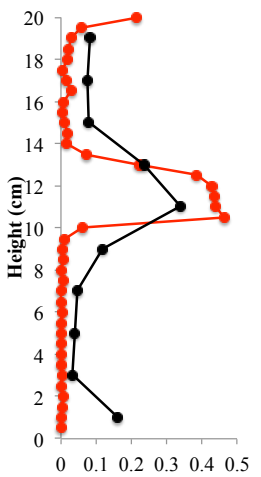

(a)

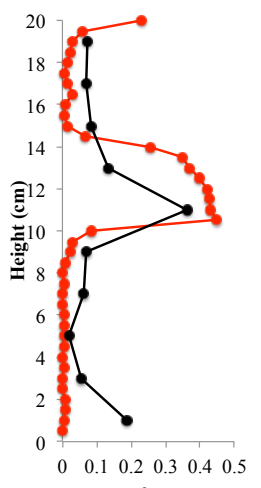

(d)

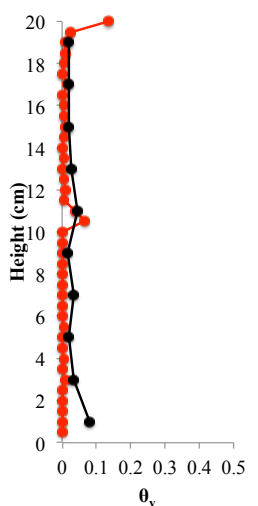

(g)

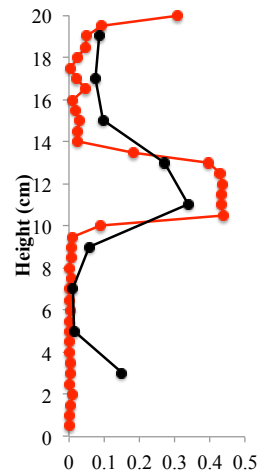

(b)

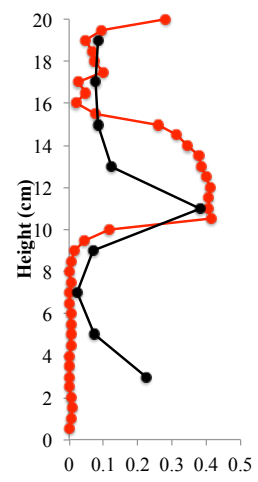

(e)

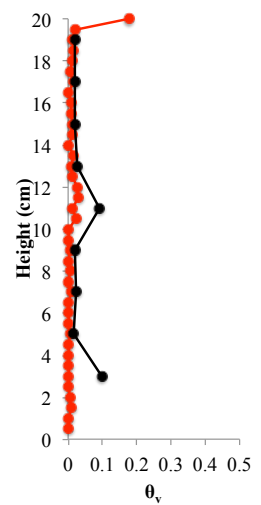

(h)

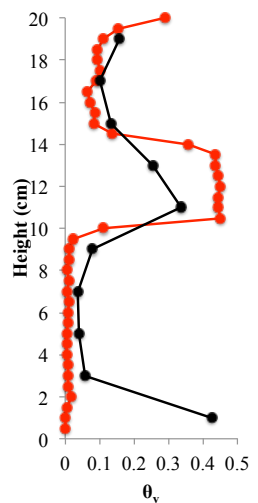

(c)

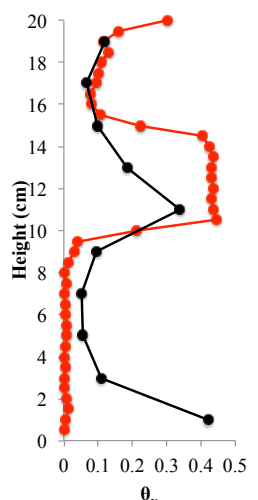

(f)

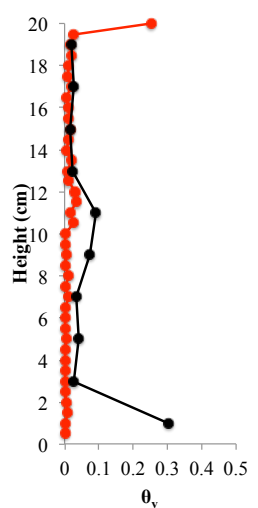

(i)
Figure 5. Profiles of volumetric water content for experiments (red line) and simulations (black line): (a) FC1, (b) FC2, (c) FC3, (d) FM1, (e) FM2, (f) FM3, (g) MC1, (h) MC2, and (i) MC3.

\section{Discussion}

\subsection{Comparison with SNOWPACK}

The SNOWPACK numerical snowpack model can also be used to reproduce dynamics observed during laboratory experiments. While Avanzi et al. (2016) compared their results with SNOWPACK-3.3.0 simulations at the end of each ex- periment (i.e. at the observed/modelled arrival time of water at the snow base), a direct comparison between models can be made for any point of time. Here, we compared temporal changes in the simulated water content profiles for SNOWPACK and the 3-D model in order to assess the role played by a simulation of preferential flow in controlling liquid water distribution in snow (Figs. 6, S1); therefore, we used both the matrix-flow multi-layer implementation of the Richards equation, RE-model (Wever et al., 2015), and a dual domain approach considering preferential flow, henceforth DDA-model (Wever et al., 2016b; Würzer et al., 2017). The resolution of SNOWPACK was set to $5 \mathrm{~mm}$ to match the resolution of the 3-D model.

In the SNOWPACK RE-model simulations, liquid water content in the upper layer gradually increased with time at all positions (Figs. 6a, S2), and the water content near the boundary was relatively large. The difference in water content between the layer interface and the upper part was underestimated when compared with experimental results, which confirms a marked spatial heterogeneity in liquid water distribution. On the other hand, 3-D simulation showed that liquid water quickly ponds at the boundary, which is consistent with the experimental observations (Figs. 6e and f, S4). Such an effect is obtained owing to preferential flow, which allowed water to move in small fingers and to reach deeper locations, even when most of the upper snow remained dry. The water ponding layer thickened until the formation of a preferential flow path in the lower layer. After preferential flow arrived at the snow base, expansion of the water ponding layer stopped. The difference in water content between the layer interface and the upper part was overestimated in comparison with the experimental observations. In the case of the SNOWPACK DDA-model (Fig. 6c and d), liquid water arrived quickly at the boundary and started to pond. Then, infiltration in matrix flow started. During ponding, infiltration into the lower layer was started in the preferential flow area with a very small water content (about $0.01 \%$ initially, and then gradually increasing). Although liquid water arrival at the snow base was faster than that in the RE-model, water ponding continued even after the liquid water arrival because the infiltration rate was too small in the lower layer. After a large amount of water ponded above the layer boundary, liquid water infiltration in matrix flow in the lower layer was started and the volume of water ponded in the upper layer started to decrease (e.g. in FM3, water content in the upper layer at $2 t$ was decreased from $5 / 3 t$; Fig. S3d). The RMSE for liquid water content profiles at (measured) arrival time are 0.107, 0.107 and 0.094 for SNOWPACK REmodel, DDA-model and 3-D model, respectively. While both SNOWPACK schemes yield the same RMSE, the 3-D model returns a slightly smaller value.

For arrival times, the 3-D and SNOWPACK DDA-model obtained greater accuracy than the SNOWPACK RE simulations (Fig. 7), which again suggests the importance of considering preferential flow. Causes of delay in 1-D models in- 


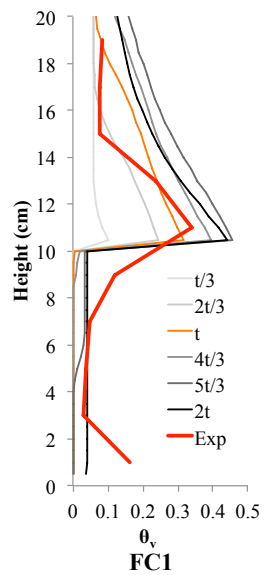

(a)

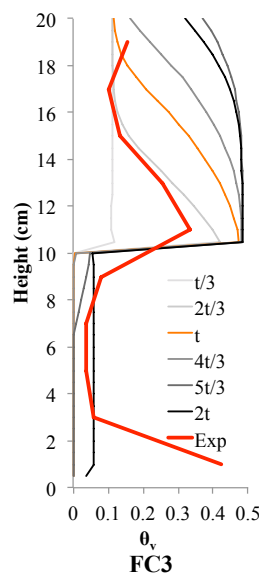

(b)

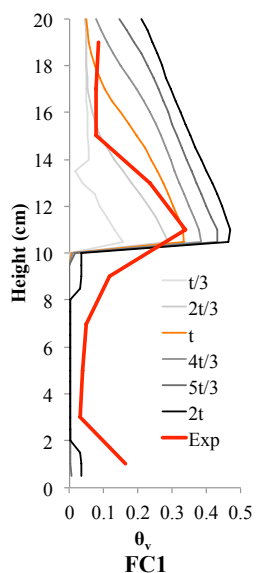

(c)

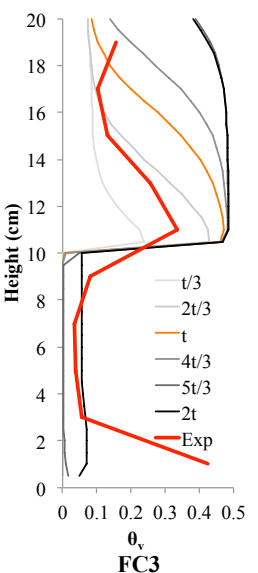

(d)

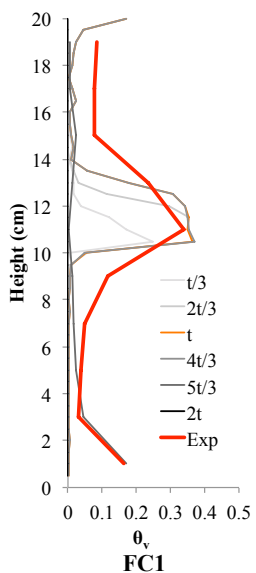

(e)

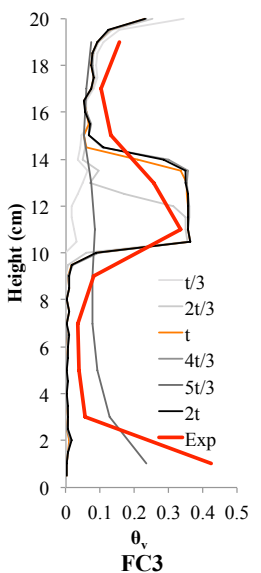

(f)

Figure 6. Temporal evolution of simulated water content profiles: (a) FC1 in SNOWPACK RE-model, (b) FC3 in SNOWPACK RE-model, (c) FC1 in SNOWPACK DDA-model, (d) FC3 in SNOWACK DDA-model, (e) FC1 in the 3-D model, and (f) FC3 in the 3-D model. Six profiles are shown in units of the arrival time at snow base in the experiment, $t$ (see Table 2).

clude both slow infiltration of matrix flow and overestimation of water ponding at the capillary barrier (Fig. 6). In terms of arrival time, delay was resolved to some degree by considering preferential flow in the SNOWPACK model. However, the SNOWPACK DDA-model is still prone to overestimating the total amount of water that ponds at the interface between layers. These comparisons between the SNOWPACK, 3-D model and laboratory experiments demonstrate the need to improve existing theories of water infiltration in snow.

The theory of water transport in the SNOWPACK REmodel is based on gravity drainage column experiments that neglect water entry suction (i.e. experiments performed using wet snow; Yamaguchi et al., 2012). In contrast, the 3-D model and SNOWPACK DDA-model include an attempt to simulate the infiltration process into initially dry snow using water entry suction (where we define dry snow as that with a lower liquid water content than the irreducible water content), which is key to reproducing fingers (Hirashima et al., 2014a). Under these conditions, the van Genuchten model could only be used with additional assumptions (Hirashima et al., 2014a). Accordingly, we assumed that dry snow had a threshold suction equal to water entry suction. Future work will focus on improving this approach; for example, water entry suction may be related to the suction-wetness profile of a wetting water retention curve (Avanzi et al., 2016), which has not yet been parameterized. Furthermore, unsaturated conductivity tends towards zero in dry conditions, but extensive observations of unsaturated conductivity in snow are missing.

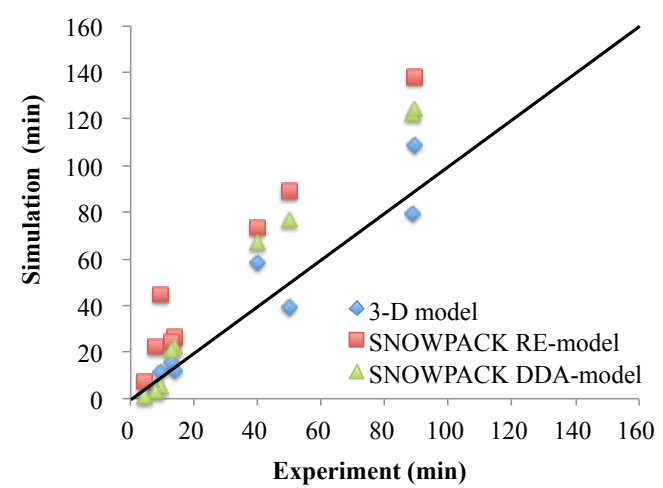

Figure 7. Comparison of water arrival between a laboratory experiment, a 3-D model and two SNOWPACK models.

\subsection{Wet snow ratio and preferential flow path area}

The main purpose of the development of this model is to better understand 3-D patterns of water infiltration in snow and, thus, resolve the delay of the arrival time as a limitation of matrix-flow models. The simulation results showed that the model can reproduce preferential flow and capillary barriers and, consequently, provide reliable estimations of the arrival time of water at the sample base. On the other hand, the model underestimated the simulated preferential flow area. In terms of effect on arrival time, this underestimation is not a serious problem because the travel time through the preferential flow area was short (Table 2); however, it may represent a problem for long-term simulations, especially when estimating the transition from a predominantly dry snow to a predominantly wet snow. 
According to the simple model of Baker and Hillel (1990), the wetted fraction of the sublayer in a finer-over-coarser transition depends on water input rate and unsaturated conductivity during steady vertical infiltration. Horizontal expansion of preferential flow also depends on infiltration along the horizontal direction. As the direction of water flow depends on gravity (vertical) and capillarity, movement in the horizontal direction may be impeded if simulated capillary gradients are small. For example, the fact that fine snow in experiments had larger preferential flow paths than coarse snow was probably due to a greater heterogeneity in capillarity in fine snow (Avanzi et al., 2016).

We performed sensitivity tests to estimate the relevance of vertical and horizontal movement for different types of snow, in which we calculated which voxel (left, right, front, back, up, down) was easiest to infiltrate from a generic voxel as a function of gravity or water entry suction. We found that the ratios of the water moving to the lower voxel were 24.3, 38.8 and $60.7 \%$ for fine, medium and coarse snow, respectively. When this ratio is large (e.g. coarse snow), water moves downward, and consequently the preferential flow path areas become small. Where there is no gravitational force, the ratio would be $16.7 \%$, while for fine snow the ratio of water moving to the lower voxel was $24.3 \%$. Nevertheless, the simulated mean wet snow area was small even for fine snow (e.g. $4.8 \%$ in $\mathrm{FC} 1$ and $22 \%$ in FC3, excluding the ponding area). As the simulated wet snow area is smaller than the measured one, this model may still underestimate the effective crosssectional area of infiltration. This will be the subject of future research.

In this model, water entry suction was used as a threshold for liquid water infiltration into dry snow. However, in the measured water absorption curve of Adachi et al. (2012), the relationship between suction and liquid water content was non-linear and hysteretic (see Sect. 4.1). This simplified condition for infiltration into dry snow may lead to an underestimation of the expansion of preferential flow. Neglecting quick metamorphism in preferential flow paths (Avanzi et al., 2017a) may represent another cause of underestimation of preferential flow path size as grain growth promotes lateral spreading of water and expansion of paths. Although this model includes grain growth following Brun et al. (1989) and Tusima (1978), modelling some specific conditions such as wet snow metamorphism at the boundaries between preferential flow paths and drier snow is still an open issue. Also, existing observations of wet snow metamorphism have been mainly performed in static conditions, which means that the coupling between grain growth and flowing water is still poorly understood. This represents a further unknown for models of liquid water in snow.

The number of preferential flow paths can also promote the expansion of the wet snow area (Schneebeli, 1995). In our model, liquid water preferred to infiltrate snow along the same path; therefore, preferential flow paths did not increase unless the amount of liquid water supply also increased.
Also, compaction by wet snow metamorphism could change the balance of force distribution and create new pathways for liquid water. This underestimation may also be related to uncertainties in the computation of unsaturated water conductivity in initially dry snow and/or in the rule used to calculate the conductivity between voxels. New techniques to measure the development of preferential flow paths can help to model these processes and further experiments in this direction are, therefore, highly needed.

\subsection{Impact of grain size on water infiltration process}

Grain size is one of the key parameters for water infiltration processes. Nevertheless, grain sizes cannot be measured at high resolution in nature (typically measured to $0.1 \mathrm{~mm}$ ); therefore, the sensitivity of model results with fluctuation in grain size is informative. In this discussion, sensitivity experiments for grain sizes fluctuating by $0.1 \mathrm{~mm}$ for the upper layer and lower layers were performed. Simulation results in terms of thickness of water ponding layer, water content profile, and arrival time are shown in Fig. 8.

The thickness of water ponding over the interface between layers is usually increased in cases of smaller upper grain size and greater lower grain size (Fig. 8a). Grain size thus significantly influences the water ponding layer. Differences in water ponding thickness between a real and decreased grain size on the upper layer ranged from 7 to $48 \%$ in the case of fine over coarse and fine over medium. On the other hand, differences for fluctuations of the lower layer were less than $8 \%$ in eight out of the nine cases (Fig. 8a), sample MC1 being the only exception (22\%). This difference suggests that the upper layer plays a pivotal role in capillary barriers. An increase in the water ponding layer in the case of decreased upper fine grains can also be clearly identified in the water content profile (Fig. 8b). Decreasing the size of upper fine grains not only increases the thickness of the water ponding layer, but also increases water content outside of the water ponding layer. For example, decreasing upper layer grain size in FC1 (Fig. 8b) yields total water contents from 15.5 to $19.5 \mathrm{~cm}$ in height that are about 2.6 times those of the original, nonfluctuating case. This reflects the increasing area of wet snow in the upper layer. The area of the preferential flow path is sensitive to both grain sizes, especially for fine snow (Katsushima et al., 2013; Hirashima et al., 2014a). Fluctuations in water content profile driven by grain size were also seen in the case of MC1, but were not significant (see Fig. 8c). Figures of water content profiles for other cases are shown in the Supplement (Fig. S5).

Variations in grain size also affect arrival time at the snow base (Fig. 8d). Differences in arrival time between increasing grain size and decreasing grain size for the upper layer were more than $50 \%$ in the cases of FC1 and FM1. These results suggest that the accuracy of measured grain size is important to estimate water infiltration, especially for fine grains. In the parameterizations for water entry suction used in our 3-D 


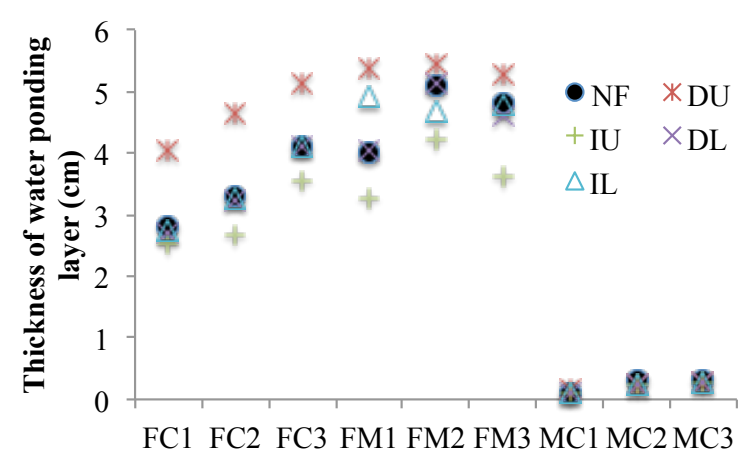

(a)

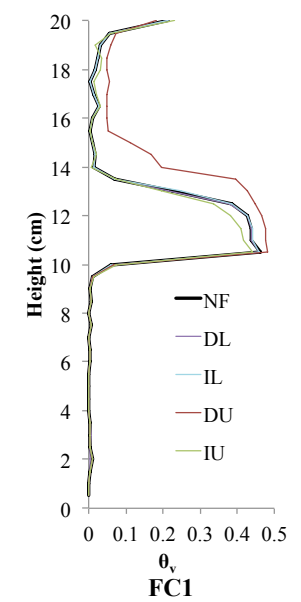

(b)

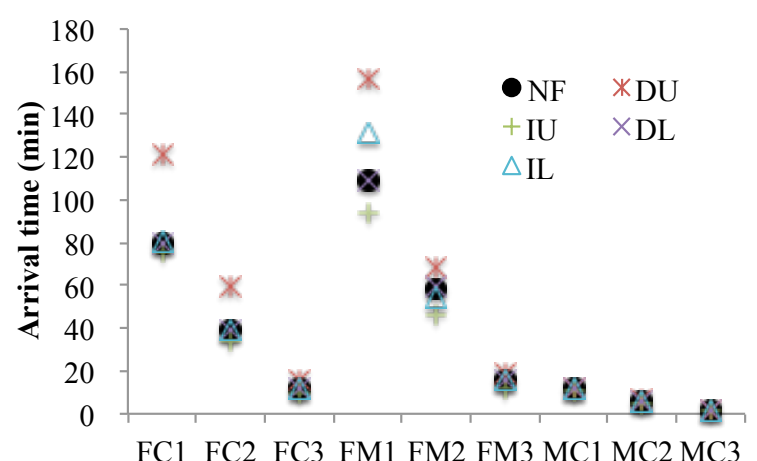

(d)

Figure 8. Influence of grain size fluctuations on the thickness of the water ponding layer (a), water content profiles $(\mathbf{b}, \mathbf{c})$ and arrival time at the snow base (d). NF: no fluctuation, IU: increase $0.1 \mathrm{~mm}$ for the upper layer, DU: decrease $0.1 \mathrm{~mm}$ for the upper layer, IL: increase $0.1 \mathrm{~mm}$ for the lower layer and DL: decrease $0.1 \mathrm{~mm}$ for the lower layer.

model and dual domain approach, grain size was determined circumstantially, providing a grain size distribution measured from individual grains. Since measuring individual grain size requires great care, methods requiring less care and the abil- ity to obtain grain size parameters with high degrees of accuracy are necessary for more accurate models. Development of measuring methods for specific surface area may improve estimation accuracy and contribute to the study of water infiltration processes.

\subsection{Outlook}

The 3-D model developed here represents an important stage in the development of an exhaustive theory of liquid water movement in snow. However, the low accuracy of preferential flow path area in our model means that it cannot be used to improve the parameterization of preferential flow area, as in Wever et al. (2016b). In the future, a thorough parameterization of hysteresis in snow and the better reconstruction of the expansion of preferential flow path area will improve the accuracy of 3-D models and allow for an advanced estimation of preferential flow area in 1-D models. Wever et al. (2016b) also suggested that 3-D models should analyse heat exchange around the preferential flow path; therefore, future developments of our model will consider heating and melt-freeze processes (e.g. the model of Leroux and Pomeroy, 2017). For this, laboratory experiments of ice layer formation will be needed for validation.

Another possible improvement to the model would be the parameterization of quick grain growth at saturation, which would be necessary for simulating the structural evolution of areas affected by ponding. Grain growth causes an abrupt decrease in suction and consequently reduces the water content at the ponding layer. The water content of the upper layer at $2 t$ was smaller than that at $5 t / 3$ (Fig. 6a) owing to a decline of suction due to grain growth. In the first version of the SNOWPACK model, the Brun et al. (1989) equation was used for estimating grain growth; however, this formula is based on data with small water content, and application to saturated conditions may overestimate grain growth. To avoid this, Hirashima et al. (2010, 2014a) used the equation of Tusima (1978) to constrain the upper limit of grain growth rate. Although this formula is based on data measured under saturated conditions, grain growth remains limited for a short timescale such as occurred during these experiments. The grain growth equation of Tusima (1978) was formulated using data for $200 \mathrm{~h}$, but it did not focus on the first $1 \mathrm{~h}$; therefore, grain growth over short time periods and under saturated conditions remains unclear. Raymond and Tusima (1979), Wakahama (1968), and Colbeck (1973) all focused on wet snow metamorphism under saturated conditions, but they did not focus on the first hour of the experiments. Extending the existing parameterizations of wet snow metamorphism for small timescales will improve simulation accuracy with regards to the development and disappearance of water ponding by capillary barriers. Also, a correct simulation of grain growth will lead to correct estimation of the lateral spreading of water, which will improve the accuracy of the prediction of preferential flow path size. 
Our results show that this model is capable of reproducing detailed water infiltration at sample scale (i.e. considering micro-scale heterogeneity). On the other hand, the intrinsic scale of this process and computational efforts mean that it is still not suitable for basin-scale simulations. This limitation could be overcome by synergies with existing physicsbased hydrologic models for snow-dominated catchments, for example, Alpine3D (Lehning et al., 2006). Currently, SNOWPACK is used as a part of Alpine3D for simulation of accumulation-ablation patterns of snowpacks. In this study, comparisons between laboratory experiments, a 3-D model, and SNOWPACK were performed and contributed to highlighting model limitations and possible avenues of future developments (e.g. an underestimation of flow path cross sections). While a 3-D model cannot reproduce the entire range of natural variability of liquid water flow in snow, it can help to replicate and understand this process in conditions that are difficult for experiments (e.g. larger sample sizes and/or a more complex stratigraphy). This may contribute to defining new parameterizations for dual domain approaches that could be then fully included in catchment-scale models. Also, we will try to apply this model at the basin scale by increasing the element size. While this will hamper the representation of single preferential fingers, we expect the model to be able to correctly reproduce other relevant features of water flow at slope scale such as lateral flow. This could help to understand liquid water flow around concave/convex portions of the landscape.

\section{Conclusions}

Validation of simulations for capillary barrier formation and subsequent preferential flow development was performed using a 3-D water transport model. Overall, the infiltration process into dry snow was well reproduced, and in particular the timing of liquid water arrival at the snow base was accurate. A detailed comparison of wet conditions in the snow column was performed to check accuracy and identify shortcomings in the model. The model accurately reproduced (a) the onset of preferential flow in initially dry snow, and (b) the ponding of liquid water above the boundary of snow layers by a capillary barrier, for which the ponded water volume was larger at the boundary of fine over coarse and fine over medium snow layers than it was at the boundary of medium over coarse snow layers.

Model discrepancies included (a) an underestimation of liquid water content and wet snow area in preferential flow path areas, and (b) overestimation of water ponding volume at the layer boundary in experiments FC and FM, but underestimation in experiment MC. Future improvements to the model will include improving the water entry process for dry snow, measurements of water content profile for capillary rise, and direct measurements of preferential flow path formation.
The advantage of this model over 1-D models is the consideration of 3-D heterogeneous infiltration into dry snow. An explicit simulation of preferential flow also returns a reliable estimation of liquid water arrival at the snow base. However, improvements are needed to ensure that the model works over both long and short time periods. An accurate reproduction of the transition from a dry-snow-dominant to wet-snow-dominant condition is an important step in upgrading this model to a full 3-D numerical snowpack model.

Data availability. The experiments performed to collect the data we used here are described in Avanzi et al. (2016). Most of the data we used are directly reported in that paper. Profiles of liquid water content and wet-snow fraction are available at https://doi.pangaea. de/10.1594/PANGAEA.882524 (Avanzi et al., 2017b).

\section{The Supplement related to this article is available online at https://doi.org/10.5194/hess-21-5503-2017- supplement.}

Competing interests. The authors declare that they have no conflict of interest.

Acknowledgements. This study was part of the "Research on Advanced Snow Information and its Application to Disaster Mitigation" project, and was supported by JSPS and KAKENHI (grant number JP15H01733). Fruitful discussions about this work with Yoshiyuki Ishii and Takafumi Katsushima are acknowledged We would like to acknowledge Nander Wever, who helped with the SNOWPACK simulations with preferential flow. We thank the members of the Snow and Ice Research Center for their advice and discussion. We are grateful to Mieko Miura, who assisted with our research. Francesco Avanzi is grateful for the support received during his visiting periods at the Snow and Ice Research Center in Nagaoka (Japan)

Edited by: Günter Blöschl

Reviewed by: two anonymous referees

\section{References}

Adachi, S., Yamaguchi, S., Ozeki, T., and Kose, K.: Hysteresis in the water retention curve of snow measured using an MRI system, Proceedings, International Snow Science Workshop, 1621 September 2012, Anchorage, Alaska, USA, 918-922, 2012.

Avanzi, F., Hirashima, H., Yamaguchi, S., Katsushima, T., and De Michele, C.: Observations of capillary barriers and preferential flow in layered snow during cold laboratory experiments, The Cryosphere, 10, 2013-2026, https://doi.org/10.5194/tc-10-20132016, 2016.

Avanzi, F., Petrucci, G., Matzl, M., Schneebeli, M., and De Michele, C.: Early formation of preferential flow in a homogeneous snow- 
pack observed by micro-CT, Water Resour. Res., 53, 3713-3729, https://doi.org/10.1002/2016WR019502, 2017a.

Avanzi, F., Hirashima, H., and Yamaguchi, S.: Profiles of liquid water content and wet-snow fraction around capillary barriers in layered snow measured during infiltration experiments in a cold laboratory, PANGAEA, https://doi.org/10.1594/PANGAEA.882524, 2017 b.

Baggi, S. and Schweizer, J.: Characteristics of wet-snow avalanche activity: 20 years of observations from a high alpine valley (Dischma, Swizerland), Nat. Hazards, 50, 97-108, 2008.

Baker, R. S. and Hillel, D.: Laboratory tests of a theory of fingering during infiltration into layered soils, Soil Sci. Soc. Am. J. 54, 20-30, 1990.

Brun, E. and Ray, L.: Field study on snow mechanical properties with special regard to liquid water content, in: Avalanche formation, movement and effects, Proceedings of the Davos Sympsium, 14-19 September 1986, Davos, Swizerland, IAHS, publ. $162,1987$.

Brun, E., Martin, E., Simon, V., Gendre, C., and Coleou, C.: An energy and mass model of snow cover suitable for operational avalanche forecasting, J. Glaciol., 35, 333-342, 1989.

Calonne, N., Geindreau, C., Flin, F., Morin, S., Lesaffre, B., Rolland du Roscoat, S., and Charrier, P.: 3-D image-based numerical computations of snow permeability: links to specific surface area, density, and microstructural anisotropy, The Cryosphere, 6 , 939-951, https://doi.org/10.5194/tc-6-939-2012, 2012.

Carmagnola, C. M., Morin, S., Lafaysse, M., Domine, F., Lesaffre, B., Lejeune, Y., Picard, G., and Arnaud, L.: Implementation and evaluation of prognostic representations of the optical diameter of snow in the SURFEX/ISBA-Crocus detailed snowpack model, The Cryosphere, 8, 417-437, https://doi.org/10.5194/tc8-417-2014, 2014.

Clifford, K. H. and Stephen, W. W.: Capillary barrier performance in heterogeneous porous media, Water Resour. Res., 34, 603609, 1998.

Colbeck, S. C.: A theory of water percolation in snow, J. Glaciol., 11, 369-385, 1972.

Colbeck, S. C.: Theory of metamorphism of wet snow, CRREL Res. Rep. 313, US Army Cold Regions Research and Engineering Laboratory, Hanover, New Hampshire, USA, 1973.

Colbeck, S. C.: The capillary effects on water percolation in homogeneous snow, J. Glaciol., 13, 85-97, 1974a.

Colbeck, S. C.: Water flow through snow overlying an impermeable boundary, Water Resour. Res., 10, 119-123, https://doi.org/10.1029/WR010i001p00119, 1974b.

Colbeck, S. C.: An analysis of water flow in dry snow, Water Resour. Res., 12, 523-527, https://doi.org/10.1029/WR012i003p00523, 1976.

Colbeck, S. C.: Theory of metamorphism of dry snow, J. Geophys. Res., 88, 5475-5482, 1983.

Colbeck, S. C. and Davidson, G.: Water percolation through homogeneous snow, in: Int. Symp. on the Role of Snow and Ice in Hydrology, 6-13 September 1972, Banff, AB, Canada, 242-257, 1972.

Daanen, R. P. and Nieber, J. L.: Model for Coupled Liquid Water Flow and Heat Transport with Phase Change in a Snowpack, J. Cold Reg. Eng., 23, 43-68, 2009.
Dunne, T., Price, A. G., and Colbeck, S. C.: The Generation of Runoff From Subarctic Snowpacks, Water Resour. Res., 12, 677685, 1976.

Eiriksson, D., Whitson, M., Luce, C. H., Marshall, H. P., Bradford, J., Benner, S. G., Black, T., Hetrick, H., and McNamara, J. P.: An evaluation of the hydrologic relevance of lateral flow in snow at hillslope and catchment scales, Hydrol. Process., 27, 640-654, https://doi.org/10.1002/hyp.9666, 2013.

Fierz, C. and Föhn, P. M. B.: Long-Term observation of the water content of an alpine snowpack, Proceedings of the International Snow Science Workshop, 30 October-3 November 1994, Snowbird, Utah, USA, 117-131, 1994.

Fierz, C., Armstrong, R. L., Durand, Y., Etchevers, P., Greene, E., McClung, D. M., Nishimura, K., Satyawali, P. K., and Sokratov, S. A.: The International Classification for Seasonal Snow on the Ground, Tech. rep., IHP-VII Technical Documents in Hydrology N 83, IACS Contribution N 1, UNESCO-IHP, Paris, France, 2009.

Hill, D. E. and Parlange. J-Y.: Wetting front instability in layered soils, Soil Sci. Soc. Am. J., 36, 697-702, 1972.

Hirashima, H., Yamaguchi, S., Sato, A., and Lehning, M.: Numerical modeling of liquid water movement through layered snow based on new measurements of the water retention curve, Cold Reg. Sci. Technol., 64, 94-103, https://doi.org/10.1016/j.coldregions.2010.09.003, 2010.

Hirashima, H., Yamaguchi, S., and Katsushima, T.: A multidimensional water transport model to reproduce preferential flow in the snowpack, International Snow Science Workshop Grenoble-Chamonix Mont-Blanc, 7-11 October 2013, Grenoble, France, 31-37, 2013.

Hirashima, H., Yamaguchi, S., and Katsushima, T.: A multidimensional water transport model to reproduce preferential flow in the snowpack, Cold Reg. Sci. Technol., 108, 80-90, 2014a.

Hirashima, H., Yamaguchi, S., and Ishii, Y.: Simulation of liquid water infiltration into layered snowpacks using multidimensional water transport model, ISSW proceedings, Banff, Canada, 48,-54, 2014b.

Illangasekare, T. H., Walter Jr., R. J., Meier, M. F., and Pfeffer, W. T.: Modeling of meltwater infiltration in subfreezing snow, Water Resour. Res., 26, 1001-1012, https://doi.org/10.1029/WR026i005p01001, 1990.

Ito, Y., Matsushita, H., Hirashima, H., Ito, Y., and Noro, T.: Change in snow strength caused by rain, Ann. Glaciol, 53, 1-5, 2012.

Jordan, R., Albert, M. R., and Brun, E.: Physical processes within the snow cover and their parameterization, in: Snow and Climate, edited by: Armstrong, R. and Brun, E., Cambridge University Press, Cambridge, UK, 12-69, 2008.

Kämpf, M., Holfelder, T., and Montenegro, H.: Identification and parameterization of flow processes in artificial capillary barriers, Water Resour. Res., 39, 1276, https://doi.org/10.1029/2002WR001860, 2003.

Katsushima, T., Yamaguchi, S., Kumakura, T., and Sato, A.: Experimental analysis of preferential flow in dry snowpack, Cold Reg. Sci. Technol., 85, 206-216, https://doi.org/10.1016/j.coldregions.2012.09.012, 2013.

Kattelmann, R.: Wet slab instability, in: Proceedings of the 1984 International Snow Science Workshop, 24-27 October 1984, Aspen, Colorado, USA, 102-108, 1984. 
Lehning, M., Bartelt, P., Brown, B., Fierz, C., and Stayawali, P.: A physical SNOWPACK model for the Swiss avalanche warning Part II. Snow microstructure, Cold Reg. Sci. Technol., 35, 147167, 2002.

Lehning, M., Völksch, I., Gustafsson, D., Nguyen, T. A., Stähli, M., and Zappa, M.: ALPINE3D: a detailed model of mountain surface processes and its application to snow hydrology, Hydrol. Process., 20, 2111-2128, 2006.

Leroux, N. R. and Pomeroy, J. W.: Modelling capillary hysteresis effects on preferential flow through melting and cold layered snowpacks, Adv. Water Resour., 107, 250-264, https://doi.org/10.1016/j.advwatres.2017.06.024, 2017.

Marsh, P.: Grain Growth in a wet arctic snow cover, Cold Reg. Sci. Technol., 14, 23-31, 1987.

Marsh, P. and Woo, M.: Meltwater movement in natural heterogeneous snow covers, Water Resour. Res., 21, 1710-1716, https://doi.org/10.1029/WR021i011p01710, 1985.

Marshall, H. P., Conway, H., and Rasmussen, L. A.: Snow densification during rain, Cold Reg. Sci. Technol., 30, 35-41, https://doi.org/10.1016/S0165-232X(99)00011-7, 1999.

Mitterer, C. and Schweizer, J.: Analysis of the snow-atmosphere energy balance during wet-snow instabilities and implications for avalanche prediction, The Cryosphere, 7, 205-216, https://doi.org/10.5194/tc-7-205-2013, 2013

Mitterer, C., Hirashima, H., and Schweizer, J.: Wet-snow instabilities: comparison of easured and modelled liquid water content and snow stratigraphy, Ann. Glaciol., 52, 201-208, 2011.

Mualem, Y.: A new model for predicting the hydraulic conductivity of unsaturated porous media, Water Resour. Res., 12, 513-522, 1976.

Raymond, C. F. and Tsushima, K.: Grain coarsening of watersaturated snow, J. Glaciol., 22, 83-105, 1979.

Schneebeli, M.: Development and stability of preferential flow paths in a layered snowpack, Biogeochemistry of seasonally snow-covered catchments, proceedings of a boulder symposium, 1-14 July 1995, Boulder, Colorado, USA, IAHS Publ., 228, 8995, 1995.

Takeuchi, Y. and Hirashima, H.: Snowpack estimations in the starting zone of large-scale snow avalanches in the Makunosawa valley, Myoko, Japan, Ann. Glaciol., 54, 19-24, 2013.

Tusima, K.: Grain coarsening of ice particles immersed in pure water, Seppyo, 40, 155-164, 1978 (in Japanese, with English abstract).

van Genuchten, M. Th.: A closed-form equation for predicting the hydraulic conductivity of unsaturated soils, Soil Sci. Soc. Am. J., 44, 892-898, 1980.
Wakahama, G.: The metamorphism of wet snow, IAHS Publ., 79, 370-379, 1968.

Waldner, P. A., Schneebeli, M., Schultze-Zimmermann, U., and Flühler, H.: Effect of snow structure on water flow and solute transport, Hydrol. Proc., 18, 1271-1290, https://doi.org/10.1002/hyp.1401, 2004.

Wankiewicz, A.: A review of water movement in snow, in: Modeling of snow cover runoff, edited by: Colbeck, S. C. and Ray, M., U.S. Army Cold Regions Research and Engineering Laboratory, Hanover, New Hampshire, USA, 1978.

Wever, N., Fierz, C., Mitterer, C., Hirashima, H., and Lehning, M.: Solving Richards Equation for snow improves snowpack meltwater runoff estimations in detailed multi-layer snowpack model, The Cryosphere, 8, 257-274, https://doi.org/10.5194/tc-8-2572014, 2014.

Wever, N., Schmid, L., Heilig, A., Eisen, O., Fierz, C., and Lehning, M.: Verification of the multi-layer SNOWPACK model with different water transport schemes, The Cryosphere, 9, 2271-2293, https://doi.org/10.5194/tc-9-2271-2015, 2015.

Wever, N., Vera Valero, C., and Fierz, C.: Assessing wet snow avalanche activity using detailed physics based snowpack simulations, Geophys. Res. Lett., 43, 5732-5740, https://doi.org/10.1002/2016GL068428, 2016a.

Wever, N., Würzer, S., Fierz, C., and Lehning, M.: Simulating ice layer formation under the presence of preferential flow in layered snowpacks, The Cryosphere, 10, 2731-2744, https://doi.org/10.5194/tc-10-2731-2016, 2016 b.

Würzer, S., Wever, N., Juras, R., Lehning, M., and Jonas, T.: Modelling liquid water transport in snow under rain-on-snow conditions - considering preferential flow, Hydrol. Earth Syst. Sci., 21 , 1741-1756, https://doi.org/10.5194/hess-21-1741-2017, 2017.

Yamaguchi, S., Katsushima, T., Sato, A., and $\mathrm{Ku}-$ makura, T.: Water retention curve of snow with different grain sizes, Cold Reg. Sci. Technol., 64, 87-93, https://doi.org/10.1016/j.coldregions.2010.05.008, 2010.

Yamaguchi, S., Watanabe, K., Katsushima, T., Sato, A., and Kumakura, T.: Dependence of the water retention curve of snow on snow characteristics, Ann. Glaciol., 53, 6-12, 2012.

Yamanoi, K. and Endo, Y.: Dependence of shear strength of snow cover on density and water content, Seppyo, 64, 443-451, 2002 (in Japanese, with English abstract). 